\title{
Evaluation of Urine Reagent Strip as a Tool for Routine Diagnosis of Maternal Urogenital Schistosomiasis at Antenatal Clinic Visit in Munyenge, South West Region, Cameroon
}

\author{
Godlove Bunda Wepnje $\left(\mathbb{D},{ }^{1}\right.$ Judith Kuoh Anchang-Kimbi $\left(\mathbb{D},{ }^{1}\right.$ Leopold Gustave Lehman, ${ }^{2}$ \\ and Helen Kuokuo Kimbi (iD) \\ ${ }^{1}$ Department of Zoology and Animal Physiology, Faculty of Science, University of Buea, P.O. Box 63, Buea, Cameroon \\ ${ }^{2}$ Department of Animal Biology, Faculty of Science, University of Douala, P.O. Box 24157, Douala, Cameroon \\ ${ }^{3}$ Department of Medical Laboratory Sciences, Faculty of Health Sciences, The University of Bamenda, P.O. Box 39, \\ Bambili, Cameroon
}

Correspondence should be addressed to Godlove Bunda Wepnje; wepnje.godlove@ubuea.cm

Received 25 September 2019; Accepted 19 November 2019; Published 6 December 2019

Academic Editor: Gilbert Lefèvre

Copyright $\odot 2019$ Godlove Bunda Wepnje et al. This is an open access article distributed under the Creative Commons Attribution License, which permits unrestricted use, distribution, and reproduction in any medium, provided the original work is properly cited.

\begin{abstract}
Urine reagent strip used in detecting microhaematuria has been recommended in pregnancy for diagnosis of urogenital schistosomiasis (UGS) during routine antenatal care (ANC). This study evaluated its sensitivity, specificity, and predictive values in the diagnosis of maternal UGS using filtration method as a reference test. We also assessed the variation in its performance in the diagnosis of UGS using multiple-sample collection. A total of 93 pregnant women reporting for first ANC clinic visit at any of the three functional health care centres (Munyenge Integrated Health Centre, Banga Annex Health Centre, and Trans African Health Centre) were enrolled and followed up for three consecutive monthly visits. Urine samples were observed microscopically for S. haematobium egg using urine filtration and screened for microhaematuria and proteinuria using urine reagent strips. Twenty-two (23.7\%) out of the 93 women were diagnosed for UGS, all of whom showed S. haematobium egg excretion during all three visits. There was a significant difference $(p<0.001)$ between the prevalence of $S$. haematobium infection and the prevalence of microhaematuria. The intensity of infection was significantly higher in microhaematuria-positive women compared with microhaematuria-negative cases. Sensitivity of reagent strip ranged from 54.5 to $59.1 \%$, while specificity was above $98.0 \%$ (range: 98.6-100\%). The measure of agreement between urine filtration and reagent strip method was substantial (0.61-0.8) irrespective of different sampling periods. Urine reagent strip is a moderately sensitive method in the detection of UGS and will most likely identify women with high egg load burden. Proper diagnosis of schistosomiasis during pregnancy is recommended.
\end{abstract}

\section{Introduction}

Maternal schistosomiasis is a public health concern in endemic countries of sub-Saharan Africa where successful and sustainable control programmes are lacking [1-5]. It is estimated that approximately 10 million women annually have the infection during pregnancy [6] where maternal urogenital schistosomiasis (UGS) is associated with serious adverse effects on pregnancy such as preterm labour, low birth weight, and maternal anaemia $[3,4,7]$. Besides pregnancy associated morbidities, female genital involvement associated with $S$. haematobium infection has been reported as a plausible risk factor for HIV acquisition [8]. Praziquantel is widely acknowledged as the most important, rapid, and cost-effective method of reducing morbidity due to schistosome infections among risk groups [9]. Management of infected pregnant women through treatment with praziquantel is recommended by WHO, but many endemic countries are reluctant to adopt this policy because of the lack of sufficient safety data $[6,10]$. However, findings from controlled trial studies continue to support the expansion treatment policies to include pregnant women $[10,11]$. Studies in Cameroon have shown that the use 
of pipe-borne water eliminates infection [5], but it is not sustainable as pregnant women in endemic areas still make contact with water through domestic and bathing activities $[4,5]$.

Schistosoma haematobium infection can be diagnosed by several approaches. The urine filtration technique using polycarbonate filter membranes is recommended as the gold standard for S. haematobium egg detection [12]. Although this method costs 0.1-0.3 US dollars (USD) per test [13], its use in resource-constrained settings is limited due to unavailability. Thus, low-cost methods such as single-ply paper towels as urine filters have been improvised to substitute for the polycarbonate filters in resource limited settings [14]. Rapid detection of UGS in infected individuals is necessary for prompt intervention through treatment. Rapid immunodiagnostic kit (lateral flow urine-CCA dipstick) for diagnosis of schistosomiasis is commercially available, but poor sensitivity for $S$. haematobium $[15,16]$ has been reported. Several studies have validated the use of urine reagent strip for the detection of microhaematuria, a proxy indicator for S. haematobium. Compared with proteinuria and leukocyturia, microhaematuria is the most widely studied indicator in endemic areas with high sensitivity to $S$. haematobium infection [17-20]. Besides, urine reagent strips are used to aid in quick mapping surveys in endemic regions as it is rapid, easy to use, and less time consuming [21, 22].

Microhaematuria may be a useful rapid diagnostic tool for schistosomiasis in pregnant women and has been recommended to be introduced in routine antenatal care in Schistosoma-endemic areas [19]. To assess the performance characteristics of urine reagent strip in diagnosis of maternal UGS, we evaluated its sensitivity, specificity, and predictive values using filtration method as a reference diagnostic test. Furthermore, we assessed the variation in the performance of reagent strip test in the diagnosis of UGS in pregnant women followed up for three consecutive monthly visits.

\section{Materials and Methods}

2.1. Study Area. The study was conducted in Munyenge, South West Region, Cameroon. As previously reported, Munyenge is mesoendemic for S. haematobium infection [23]. This work was a prospective cohort study and part of a larger epidemiological study on maternal urogenital schistosomiasis conducted between November 2016 and January 2018 [5].

2.2. Study Population and Design. Pregnant women reporting for the first ANC clinic visit at any of the three functional health care centres (Munyenge Integrated Health Centre, Banga Annex Health Centre, and Trans African Health Centre) were enrolled and followed up for three consecutive monthly visits.

Inclusion criteria. Pregnant women who had lived in the area for at least six months and gave written informed consent were included in the study.

Exclusion criteria. Nonregular participants and visitors were not eligible.
2.2.1. Sample Size Determination. The sample size for the study was calculated using the formula $n=Z_{\alpha / 2}^{2}$ Se $(1-\mathrm{Se}) /$ $d^{2} \times$ Prev [24] where $n=$ sample size, $Z_{\alpha / 2}=1.96$ : confidence level test statistic at the desired level of significance, $\mathrm{Se}=98.8 \%$ : sensitivity of previous published data [25], Prev $=46.8 \%$ : prevalence of maternal UGS [4], and $d=0.05$ : acceptable error willing to be committed. The minimum sample size was estimated as $n=39$. The sample size was then increased by $40 \%$ to a minimum of 93 participants who were followed up for three ANC visits.

2.3. Rapid Diagnostic Test and Parasitological Examination. Collection and examination of urine samples were done at the laboratory unit of the health facility. During the monthly visits, enrolled study participants were provided with dry, clean, and screw capped $20 \mathrm{~mL}$ universal bottles and instructed to collect midstream urine between 10 am and 2 pm. Urine reagent strips (Mission ${ }^{\circledR}, \mathrm{ACON}$ Laboratories, Inc., USA) were used to screen for microhaematuria and proteinuria. To ensure valid results, strips were stored under required temperature $\left(2^{\circ} \mathrm{C}\right.$ to $\left.30^{\circ} \mathrm{C}\right)$. Each lot of reagent strip was checked for quality control by comparing with known negative and positive controls. The urinalysis was performed according to the manufacturer's instructions.

The presence and number of S. haematobium eggs were detected under a microscope using the urine filtration method with polycarbonate filters (Sterlitech, Kent, WA, United States of America) [26]. Briefly, $10 \mathrm{ml}$ of urine was filtered through a nylon filter and examined microscopically for schistosome eggs. Eggs were graded into light infection (1-49 eggs/10 $\mathrm{ml}$ urine) and heavy infection ( $\geq 50$ eggs $/ 10 \mathrm{ml}$ urine) [27]. Any negative slide was confirmed by a second microscopist.

\subsubsection{Outcome Variables}

(i) Microhaematuria-positive was defined as a urine sample that had a trace or positive reagent strip colour reaction $(+,++,+++)$.

(ii) Proteinuria-positive was defined as a urine sample that had a trace or positive reagent strip colour reaction $(+,++,+++)$.

(iii) S. haematobium-positive was defined as a urine filtration slide that contained at least one $S$. haematobium egg.

2.4. Statistical Analysis. Data collected was validated and analysed using SPSS version 20.0 (SPSS Inc., Chicago, USA). Data was grouped into first, second, and third ANC visit. The statistical tests performed included Pearson's chi-square for comparison of proportions and the Mann-Whitney and Kruskal-Wallis tests to determine significant differences in the S. haematobium egg intensity. Diagnostic performance of urine reagent strip was determined by calculating sensitivity, specificity, positive and negative predictive values, and accuracy. Using the urine filtration method as the gold standard, sensitivity of urine reagent strip was calculated as 
TABLE 1: Characteristics of study participants.

\begin{tabular}{|c|c|c|c|}
\hline Variable & Category & Number examined $(N)$ & $\%$ \\
\hline \multirow{3}{*}{ Age group (years) } & $\leq 20$ & 36 & 38.7 \\
\hline & $21-25$ & 34 & 36.6 \\
\hline & $>25$ & 23 & 24.7 \\
\hline \multirow{2}{*}{ Marital status } & Single & 34 & 36.6 \\
\hline & Married & 59 & 63.4 \\
\hline \multirow{2}{*}{ Level of education } & At least primary & 25 & 26.9 \\
\hline & At least secondary & 68 & 73.1 \\
\hline \multirow{4}{*}{ Occupation } & Housewife & 9 & 9.7 \\
\hline & Business & 28 & 30.1 \\
\hline & Student & 33 & 35.5 \\
\hline & Farmer & 23 & 24.7 \\
\hline \multirow{2}{*}{ Stream usage } & Yes & 71 & 76.3 \\
\hline & No & 22 & 23.7 \\
\hline \multirow{2}{*}{ Stream activity } & Domestic contact + bathing & 18 & 25.4 \\
\hline & Domestic contact only & 53 & 74.6 \\
\hline \multirow{2}{*}{ Stream visits per week } & $<3$ times & 47 & 66.2 \\
\hline & $\geq 3$ times & 24 & 33.8 \\
\hline \multirow{2}{*}{ Access to piped-borne water } & Yes & 23 & 24.7 \\
\hline & No & 70 & 75.3 \\
\hline
\end{tabular}

the proportion of positives that were correctly identified when compared with the standard test. The specificity of the test was calculated as the proportion of negatives that were correctly identified when compared with the diagnostic reference test. Positive predictive value was the probability that a disease is present when the test is positive while negative predictive value was the probability that the disease is not present when the test is negative. Accuracy was the overall probability that a patient will be correctly classified. $p$ values $<0.05$ were considered statistically significant.

The agreement between the urine filtration versus reagent strip was determined using kappa $(\kappa)$-statistics. The $\kappa$-statistics were interpreted as follows: <0.0, $0.0-0.2$, $0.21-0.4,0.41-0.6,0.61-0.8,0.81-0.99$, and 1.0 , indicating no agreement, slight agreement, fair agreement, moderate agreement, substantial agreement, almost perfect agreement, and perfect agreement, respectively [28].

2.5. Ethics Approval and Consent to Participate. Ethical clearance (No2017/0481/UB/FHS/IRB) was obtained from the University of Buea, Faculty of Health Sciences Institutional Review Board, and administrative authorisation from the South West Regional Delegation of Public Health, Buea and District Medical Officer for Muyuka Subdivision. Written and verbal informed consent was obtained before enrolment into the study. Participation was voluntary and study participants were assured of confidentiality and anonymity of data.

\section{Results}

A total of 93 pregnant women were enrolled and followed up for three months. The mean age of pregnant women was $23 \pm 4.9$ years (range: $15-38$ ). The distribution of sociodemographic and stream behaviour characteristics of study participants is presented in Table 1. Generally, women made fewer visits ( $<3$ times/week) to the stream. Nonetheless, women who reported stream contact carried out one or two stream activities (domestic or bathing). Most women had no access to piped-borne water (Table 1).

Pregnant women were screened for S. haematobium infection, microhaematuria, and proteinuria for three consecutive monthly visits. The prevalence of infection due to S. haematobium and morbidity indicators did not vary between visits (Table 2). There was a significant difference $(p<0.001)$ between $S$. haematobium infection and microhaematuria. However, no variation was seen across the different visits. No association was recorded between infection status and proteinuria except for the first visit which showed marginal significance $(p=0.049)$ (Table 3$)$. The intensity of infection (egg counts) was significantly higher in microhaematuria-positive women compared with microhaematuria-negative cases. The association was similar across the visits (Table 3 ).

The sensitivity (detection of $S$. haematobium-positive cases) and specificity (detection of negative cases) of microhaematuria and proteinuria in the diagnosis of $S$. haematobium infection are shown in Table 4. Screening for microhaematuria alone enabled accurate detection of more than 50\% (range: 54.5-59.1) of S. haematobium-positive cases and above $98.0 \%$ (range: $98.6-100$ ) of negative cases. PPV was above $92.0 \%$ (range: 92.3-100) (Table 3). The sensitivity of proteinuria was consistently low (0-13.6\%) in detecting $S$. haematobium-positive cases. The measure of agreement between urine filtration method and the urine reagent strip was substantial (0.61-0.8) across visits.

\section{Discussion}

This study evaluated the performance characteristics of urine reagent strip in the diagnosis of maternal UGS at routine antenatal care. Furthermore, we assessed the variation in its 
TABLe 2: Prevalence of S. haematobium infection, microhaematuria, proteinuria, infection intensity, and mean egg load/10 ml of urine stratified by ANC visits.

\begin{tabular}{|c|c|c|c|c|c|}
\hline Outcome & Category & $1^{\text {st }}$ ANC visit $\%(n)$ & $2^{\text {nd }}$ ANC visit $\%(n)$ & $3^{\text {rd }}$ ANC visit $\%(n)$ & $\chi 2 ; p$ value \\
\hline \multirow{2}{*}{ S. haematobium infection status } & Positive & $23.7(22)$ & $23.7(22)$ & $23.7(22)$ & \multirow[t]{2}{*}{$0.00 ; 1.0$} \\
\hline & Negative & $33.3(71)$ & $33.3(71)$ & $33.3(71)$ & \\
\hline \multirow{2}{*}{ Microhaematuria } & Positive & $14.0(13)$ & $14.0(13)$ & $15.1(14)$ & \multirow[t]{2}{*}{$0.06 ; 0.971$} \\
\hline & Negative & $86.0(80)$ & $86.0(80)$ & $84.9(79)$ & \\
\hline \multirow{2}{*}{ Proteinuria } & Positive & $5.4(5)$ & $1.1(1)$ & $4.3(4)$ & \multirow[t]{2}{*}{$2.70 ; 0.260$} \\
\hline & Negative & $94.6(88)$ & $98.9(92)$ & $95.7(89)$ & \\
\hline \multirow{2}{*}{ Infection intensity } & High & $27.3(6)$ & $18.2(4)$ & $22.7(5)$ & \multirow[t]{2}{*}{$0.52 ; 0.772$} \\
\hline & Low & $72.7(16)$ & $81.8(18)$ & $77.3(17)$ & \\
\hline \multicolumn{2}{|c|}{ Mean $( \pm S D)$ egg load $/ 10 \mathrm{ml}$ of urine } & $38.5(52.6)$ & $28.3(33.1)$ & $27.0(26.6)$ & *1.93; 0.147 \\
\hline
\end{tabular}

${ }^{*}=$ Kruskal-Wallis test $(\mathrm{H})$.

TAвLE 3: Association between S. haematobium egg infection status and infection intensity, proteinuria, and microhaematuria. Association between S. haematobium infection status, microhaematuria, and proteinuria

\begin{tabular}{|c|c|c|c|c|c|c|}
\hline \multirow[t]{2}{*}{ ANC visit } & \multirow[t]{2}{*}{ Indicators } & & \multirow[t]{2}{*}{ Number } & \multicolumn{2}{|c|}{$\begin{array}{c}\text { S. haematobium infection } \\
\text { status }\end{array}$} & \multirow[t]{2}{*}{${ }^{\#} p$ value } \\
\hline & & & & Pos \% $(n)$ & Neg \% $(n)$ & \\
\hline \multirow{4}{*}{$1^{\text {st }}$ visit } & \multirow{2}{*}{ Microhaematuia } & Pos & 13 & $92.3(12)$ & $7.7(1)$ & \multirow{2}{*}{$<0.001$} \\
\hline & & Neg & 80 & $12.5(10)$ & $87.5(70)$ & \\
\hline & \multirow{2}{*}{ Proteinuria } & Pos & 5 & $60.0(3)$ & $40.0(2)$ & \multirow{2}{*}{0.049} \\
\hline & & Neg & 88 & $21.6(19)$ & $78.4(69)$ & \\
\hline \multirow{4}{*}{$2^{\text {nd }}$ visit } & \multirow{2}{*}{ Microhaematuria } & Pos & 13 & $100.0(13)$ & $0.0(0)$ & \multirow{2}{*}{$<0.001$} \\
\hline & & Neg & 80 & $11.2(9)$ & $88.8(71)$ & \\
\hline & \multirow{2}{*}{ Proteinuria } & Pos & 1 & $0.0(0)$ & $100.0(1)$ & \multirow{2}{*}{0.576} \\
\hline & & Neg & 92 & $23.9(22)$ & $76.1(70)$ & \\
\hline \multirow{4}{*}{$3^{\text {rd }}$ visit } & \multirow{2}{*}{ Microhaematuria } & Pos & 14 & $92.9(13)$ & $7.1(1)$ & \multirow{2}{*}{$<0.001$} \\
\hline & & Neg & 79 & $11.4(9)$ & $88.6(70)$ & \\
\hline & \multirow{2}{*}{ Proteinuria } & Pos & 4 & $0.0(0)$ & $100.0(4)$ & \multirow[b]{2}{*}{0.255} \\
\hline & & Neg & 89 & $24.7(22)$ & $75.3(67)$ & \\
\hline \multicolumn{7}{|c|}{ Association between infection intensity and microhaematuria } \\
\hline ANC visit & Status & & $N$ & \multicolumn{2}{|c|}{$\begin{array}{c}\text { Mean }( \pm \mathrm{SD}) \text { egg load } / 10 \mathrm{ml} \\
\text { urine (range) }\end{array}$} & ${ }^{\&} p$ value \\
\hline \multirow{2}{*}{$1^{\text {st }}$ visit } & Eggs/microhaematuria & & \multirow{2}{*}{$\begin{array}{l}12 \\
10\end{array}$} & \multicolumn{2}{|c|}{$61.2(62.1)$} & \multirow{2}{*}{0.004} \\
\hline & Eggs/no microhaematuria & & & \multicolumn{2}{|c|}{$11.4(15.7)$} & \\
\hline \multirow{2}{*}{$2^{\text {nd }}$ visit } & Eggs/microhaematuria & & 13 & \multirow{2}{*}{\multicolumn{2}{|c|}{$\begin{aligned} 41.6 & (37.6) \\
9.1 & (7.3)\end{aligned}$}} & \multirow{2}{*}{$<0.001$} \\
\hline & Eggs/no microhaematuria & & 9 & & & \\
\hline \multirow{2}{*}{$3^{\text {rd }}$ visit } & Eggs/microhaematuria & & 13 & & & \\
\hline & Eggs/no microhaematuria & & 9 & & & 0.017 \\
\hline
\end{tabular}

${ }^{\#}=$ Chi-square test, ${ }^{\&}=$ Mann-Whitney $U$ test, Pos = positive, Neg = negative.

performance using multiple urine test samples at different collection periods.

Our study clearly showed that microhaematuria was significantly associated with maternal urogenital schistosomiasis which suggests that it is a good biomarker for infection in pregnant women in endemic areas. This is in line with reports by other authors [19, 29-31]. Schistosoma haematobium-associated microhaematuria results from bladder lesions that result from the deposition of the parasite's spiny eggs in the submucosa [30]. Inconclusive evidence has suggested S. haematobium-associated glomeruli pathology leading to proteinuria $[17,19,32,33]$. Contrarily, our study did not show association between the occurrence of proteinuria and S. haematobium infection among pregnant women. However, proteinuria has other causes that are unrelated to $S$. haematobium infection which include genitourinary infection and/or the sequelae of genital mutilation [33] and preeclampsia [19]. Hence, a suspected UGSinduced proteinuria by urine reagent strip in pregnant women must be confirmed by microscopy. The association between egg load and microhaematuria has been reported $[25,34]$ with high intensity of $S$. haematobium egg excretion significantly associated with higher odds to present with microhaematuria [22]. Our findings support this association as women who shed eggs with detectable microhaematuria had significantly high egg load excretion.

The sensitivity of microhaematuria (range: 54.5-59.1\%) to detect infected individuals in our study was similar to that (61.1\%) reported in a larger survey in the same study area [5]. Similarly, moderate sensitivity of microhaematuria has been reported in other parts of Schistosoma-endemic regions of Africa [22, 35] although some studies have recorded higher 
TABle 4: Performance of microhaematuria and proteinuria in the diagnosis of UGS among pregnant women.

\begin{tabular}{|c|c|c|c|}
\hline ANC visit & Diagnostic parameters & Microhaematuria (95\% CI) & Proteinuria $(95 \% \mathrm{CI})$ \\
\hline \multirow{6}{*}{$1^{\text {st }}$ visit } & Sensitivity & $54.5(34.6-73.1)$ & $13.6(4.7-33.3)$ \\
\hline & Specificity & $98.6(92.4-99.7)$ & $97.2(90.3-99.2)$ \\
\hline & PPV & $92.3(66.7-98.6)$ & $60.0(23.1-88.2)$ \\
\hline & NPV & $87.5(78.5-93.1)$ & $78.4(68.7-85.7)$ \\
\hline & Accuracy & $88.2(80.0-93.3)$ & $77.4(67.9-84.7)$ \\
\hline & $\kappa$ value & 0.6 & 0.1 \\
\hline \multirow{6}{*}{$2^{\text {nd }}$ visit } & Sensitivity & $59.1(38.7-76.7)$ & $0.0(-)$ \\
\hline & Specificity & $100.0(94.9-100.0)$ & $98.6(92.4-99.7)$ \\
\hline & PPV & $100.0(77.2-100.0)$ & $0.0(-)$ \\
\hline & NPV & $88.7(80.0-94.0)$ & $76.1(66.4-83.6)$ \\
\hline & Accuracy & $90.3(82.6-94.8)$ & $75.3(65.6-82.6)$ \\
\hline & $\kappa$ value & 0.7 & -0.02 \\
\hline \multirow{6}{*}{$3^{\text {rd }}$ visit } & Sensitivity & $59.1(38.7-76.7)$ & $0.0(-)$ \\
\hline & Specificity & $98.6(92.4-99.7)$ & $94.4(86.4-97.8)$ \\
\hline & PPV & $92.9(68.5-98.7)$ & $0.0(-)$ \\
\hline & NPV & $88.6(79.7-93.9)$ & $75.3(65.4-83.1)$ \\
\hline & Accuracy & $89.2(81.3-94.0)$ & $72.0(62.2-88.1)$ \\
\hline & $\kappa$ value & 0.7 & -0.08 \\
\hline
\end{tabular}

$\mathrm{PPV}=$ positive predictive value; $\mathrm{NPV}=$ negative predictive value.

sensitivity values (range: $82-98 \%$ ) [19, 20, 25]. Knopp et al. [22] suggested that high intensity of infection may influence accuracy of reagent strip results. Likewise, the association between microhaematuria and egg intensity observed in the present study suggests that the reagent strip method may correctly identify cases with high egg load burden.

PPV was very high among pregnant women indicating that a greater fraction of microhaematuria in such setting is related to schistosomiasis. NPV and PPV are well known to be prevalence dependent; the higher the prevalence of the disease in a given setting, the higher the PPV and the lower the NPV $[36,37]$. In this respect, our findings on the NPV and PPV imply that a positive reagent strip test would necessarily relate to a positive $S$. haematobium infection. Comparing the results of urine reagent strip and filtration, we found that the agreement between both methods was substantial. Consequently, reagent strips, often used to monitor medical conditions of pregnant women during antenatal care, could become a very useful tool for quick diagnosis of schistosomiasis for rational control measure.

It is generally recommended that testing for schistosomiasis be repeated by follow-up with two or more consecutive visits for increased accuracy [38]. In this present study, we demonstrated stability in egg excretion among pregnant women irrespective of the different sampling periods.

\section{Conclusion}

Urine reagent strip is a moderately sensitive method in the detection of maternal UGS. Sampling at different time periods did not influence diagnostic performance of urine reagent strip. Routine urinalysis will most likely identify women with high infection burden; thus, proper diagnosis and management of schistosomiasis during pregnancy is recommended to forestall S. haematobium-induced adverse pregnancy outcomes and reduce transmission.

\section{Abbreviations}

UGS: Urogenital schistosomiasis

ANC: Antenatal care clinic.

\section{Data Availability}

All datasets used to support the findings of this study are included within the article.

\section{Conflicts of Interest}

The authors declare that they have no conflicts of interest.

\section{Acknowledgments}

The authors are grateful to all the pregnant women who gave their consent to participate in the study. Our special thanks to the Chief of Centre, nurses, and laboratory technicians of the Munyenge Integrated Health Centre, Banga Annex Health Centre, and Trans African Health Services for their cooperation and contribution. We are heartily thankful to Dr. Jodie Dionne-Odom and Miss. Renata Hocking from the University of Alabama at Birmingham, USA, for the provision of kits for the filtration method. This work was supported by the special fund for research and modernization given to the authors by the Government of Cameroon.

\section{References}

[1] A. Ajanga, N. J. Lwambo, I. Blair, U. Nyandindi, A. Fenwick, and S. Brooker, "Schistosoma mansoni in pregnancy and association with anaemia in Northwest Tanzania," Transactions of Royal Society for Tropical Medicine and Hygiene, vol. 100, no. 1, pp. 59-63, 2012.

[2] O. T. Salawu and A. B. Odaibo, "Schistosomiasis among pregnant women in rural communities in Nigeria," International Journal of Gynecology \& Obstetrics, vol. 122, no. 1, pp. 1-4, 2013. 
[3] G. Mombo-Ngoma, J. Honkpehedji, A. Basra et al., "Urogenital schistosomiasis during pregnancy is associated with low birth delivery: analysis of a prospective cohort of pregnant women and their offspring in Gabon," International Journal of Parasitology, vol. 47, no. 1, pp. 69-74, 2016.

[4] J. Anchang-Kimbi, D. E. Mansoh, G. T. Sotoing, and E. A. Achidi, "Coinfection with Schistosoma haematobium and Plasmodium falciparum and anaemia severity among pregnant women in Munyenge, Mount Cameroon area: a cross-sectional study," Journal of Parasitology Research, vol. 2017, Article ID 6173465, 12 pages, 2017.

[5] G. B. Wepnje, J. K. Anchang-Kimbi, V. D. Ndassi, L. G. Lehman, and H. K. Kimbi, "Schistosoma haematobium infection status and its associated risk factors among pregnant women in Munyenge, South West Region, Cameroon following scale-up of communal piped water sources from 2014 to 2017: a cross-sectional study," BMC Public Health, vol. 19, no. 1, p. 392, 2019.

[6] World Health Organization, Report of the WHO Informal Consultation on the Use of Praziquantel during Pregnancy/ Lactation and Albendazole/Mebendazole in Children under 24 Months, World Health Organization, Geneva, Switzerland, 2003.

[7] D. Siegrist and P. Siegrist-Obimpeh, "Schistosoma haematobium infection in pregnancy," Acta Tropica, vol. 50, no. 4, pp. 317-321, 1992.

[8] P. S. Mbabazi, O. Andan, D. W. Fitzgerald, L. Chitsulo, D. Engels, and J. A. Downs, "Examining the relationship between urogenital schistosomiasis and HIV infection," PLoS Neglected Tropical Diseases, vol. 5, no. 12, 2011.

[9] C. Ming-Gang, "Use of praziquantel for clinical treatment and morbidity control of schistosomiasis japonica in China: a review of 30 years' experience," Acta Tropica, vol. 96, no. 2-3, pp. 168-176, 2005.

[10] J. F. Friedman, R. M. Olveda, M. H. Mirochnick, A. L. Bustinduy, and A. M. Elliottd, "Praziquantel for the treatment of schistosomiasis during human pregnancy," Bulletin of the World Health Organization, vol. 96, no. 1, pp. 59-65, 2017.

[11] R. M. Olveda, L. P. Acosta, V. Tallo et al., "Efficacy and safety of praziquantel for the treatment of human schistosomiasis during pregnancy: a phase 2, randomised, double-blind, placebo-controlled trial," The Lancet Infectious Diseases, vol. 16, no. 2, pp. 199-208, 2016.

[12] WHO, Preventive Chemotherapy in Human Helminthiasis: Coordinated Use of Anthelminthic Drugs in Control Interventions: A Manual for Health Professionals and Programme Managers, World Health Organization, Geneva, Switzerland, 2006.

[13] J. Utzinger, S. L. Becker, L. van Lieshout, G. J. van Dam, and S. Knopp, "New diagnostic tools in schistosomiasis," Clinical Microbiology and Infection, vol. 21, no. 6, pp. 529-542, 2015.

[14] R. K. D. Ephraim, E. Duah, J. R. Andrews, and I. I. Bogoch, "Ultra-low-cost urine filtration for schistosoma haematobium diagnosis: a proof-of-concept study," The American Journal of Tropical Medicine and Hygiene, vol. 91, no. 3, pp. 544-546, 2014.

[15] B. Ayele, B. Erko, M. Legesse, A. Hailu, and G. Medhin, "Evaluation of circulating cathodic antigen (CCA) strip for diagnosis of urinary schistosomiasis in Hassoba school children, Afar, Ethiopia," Parasite, vol. 15, no. 1, pp. 69-75, 2008.

[16] L. A. Tchuem Tchuenté, C. J. Kueté Fouodo, and R. I. Kamwa Ngassamet al., "Evaluation of circulating cathodic antigen
(CCA) urine-tests for diagnosis of Schistosoma mansoni infection in Cameroon," PLoS Neglected Tropical Diseases, vol. 6, no. 3, Article ID e1758, 2012.

[17] O. Morenikeji, J. Quazim, C. Omoregie et al., "A cross-sectional study on urogenital schistosomiasis in children; haematuria and proteinuria as diagnostic indicators in an endemic rural area of Nigeria," African Health Sciences, vol. 14, no. 2, pp. 390-396, 2014.

[18] E. A. Ochodo, G. Gopalakrishna, B. Spek et al., "Circulating antigen tests and urine reagent strips for diagnosis of active schistosomiasis in endemic areas," Cochrane Database Systematic Reviews, vol. 3, 2015.

[19] O. T. Oyeyemi and A. B. Odaibo, "Maternal urogenital schistosomiasis; monitoring disease morbidityby simple reagent strips," PLoS One, vol. 12, no. 11, Article ID e0187433, 2017.

[20] O. B. Awosolu, F. P. Adesina, O. S. Eke, and O. J. Akinnifesi, "Efficacy of chemical reagent strip in the diagnosis of urinary schistosomiasis in Ikota, Ifedore local government area, Ondo State, Nigeria," Journal of Bacteriology and Parasitology, vol. 10, no. 2, p. 354, 2019.

[21] S. Brooker and J. Utzinger, "Integrated disease mapping in a polyparasitic world," Geos Health, vol. 1, no. 2, p. 141e6, 2007.

[22] S. Knopp, S. M. Ame, J. Hattendorf et al., "Urogenital schistosomiasis elimination in Zanzibar: accuracy of urine filtration and haematuria reagent strips for diagnosing light intensity Schistosoma haematobium infections," Parasites \& Vectors, vol. 11, no. 1, p. 552, 2018.

[23] H. N. Ntonifor, A. E. Green, M. O. S. Bopda, and J. T. Tabot, "Epidemiology of urogenital schistosomiasis and soil transmitted helminthiasis in a recently established focus behind Mount Cameroon," International Journal of Current Microbiology and Applied Sciences, vol. 4, no. 3, pp. 1056-1066, 2015.

[24] N. M. F. Buderer, "Statistical methodology: I. Incorporating the prevalence of disease into the sample size calculation for sensitivity and specificity," Academic Emergency Medicine, vol. 3, no. 9, pp. 895-900, 1996.

[25] K.J. Ndamukong, M. A. Ayuk, J. S. Dinga, T. N. Akenji, V. A. Ndiforchu, and V. P. Titanji, "Infection pattern of Schistosoma haematobium in primary school children of the Kumba health District, South-West Cameroon," African Journal of Health Sciences, vol. 7, no. 3-4, pp. 98-102, 2000.

[26] N. O. Christensen, G. Gotsche, and F. Frandsen, "Parasitological techniques for use in routine laboratory maintenance of schistosomes and used in studies on the epidemiology of human and bovine schistosomiasis," Danish Bilhaziasis Laboratory Manual, p. 112, 1984.

[27] WHO, Report of the WHO Informal Consultation on the Use of Praziquantel during Pregnancy/Lactation and Albendazolel Mebendazole in Children under 24 months, vol. 4, WHO, Geneva, Switzerland, 2002.

[28] J. R. Landis and G. G. Koch, "The measurement of observer agreement for categorical data," Biometrics, vol. 33, no. 1, pp. 159-174, 1977.

[29] A. A. Fatiregun, K. O. Osungbade, and E. A. Olumide, "Diagnostic performance of screening methods for urinary schistosomiasis in a school-based control programme, in Ibadan, Nigeria," Journal of Community Medicine and Primary Health Care, vol. 17, no. 1, pp. 24-27, 2005.

[30] L. Meurs, M. Mbow, K. Vereecken, J. Menten, S. Mboup, and K. Polman, "Bladder morbidity and hepatic fibrosis in mixed Schistosoma haematobium and S. mansoni infections: a population-wide study in Northern Senegal," PLoS Neglected Tropical Disease, vol. 6, no. 9, Article ID e1829, 2012. 
[31] S. J. Krauth, H. Greter, K. Stete et al., "All that is blood is not schistosomiasis: experiences with reagent strip testing for urogenital schistosomiasis with special consideration to verylow prevalence settings," Parasites \& Vectors, vol. 8, no. 1, pp. 584-590, 2015.

[32] E. U. Chidozie and S. Y. Daniyan, "Urinary schistosomiasis epidemiological survey of urinary schistosomiasis among children in selected schools: a preliminary study in Minna, Nigeria," African Journal of Biotechnology, vol. 7, no. 16, pp. 2773-2776, 2008.

[33] G. Poggensee, I. Krantz, I. Kiwelu, and H. Feldmeier, "Screening of Tanzanian women of child-bearing age for urinary schistosomiasis: validity of urine reagent strip readings and self-reported symptoms," Bulletin of World Health Organisation, vol. 78, no. 4, pp. 542-548, 2000.

[34] E. Emukah, J. Gutman, J. Eguagie et al., "Urine heme dipsticks are useful in monitoring the impact of praziquantel treatment on Schistosoma haematobium in sentinel communities of Delta State, Nigeria," Acta Tropica, vol. 122, no. 1, pp. 126-131, 2012.

[35] O. T. Salawu and A. B. Odaibo, "Urogenital schistosomiasis and urological assessment of hematuria in preschool-aged children in rural communities of Nigeria," Journal of Pediatric Urology, vol. 10, no. 1, pp. 88-93, 2014.

[36] D. G. Altman and J. M. Bland, "Statistics notes: diagnostic tests 2: predictive values," BMJ, vol. 309 , no. 6947 , p. 102 , 1994.

[37] T. F. Heston, "Standardizing predictive values in diagnostic imaging research," Journal of Magnetic Resonance Imaging, vol. 33, no. 2, p. 505, 2011.

[38] K. G. A. D. Weerakoon, G. N. Gobert, P. Cai, and D. P. McManus, "Advances in the diagnosis of human Schistosomiasis," Clinical Microbiology Reviews, vol. 28, no. 4, pp. 939-967, 2015. 


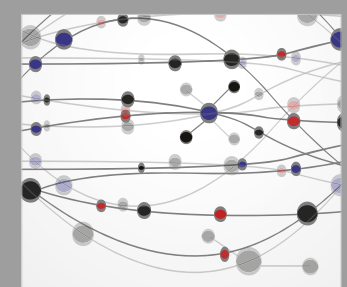

The Scientific World Journal
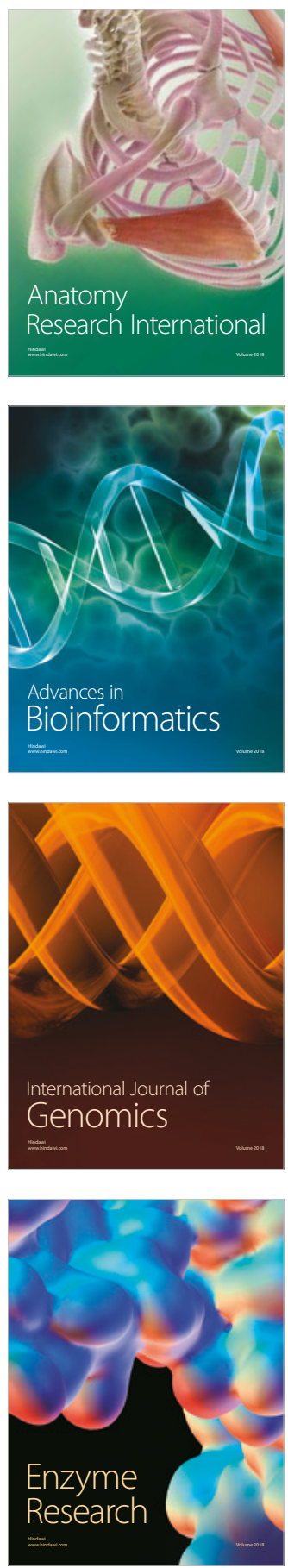
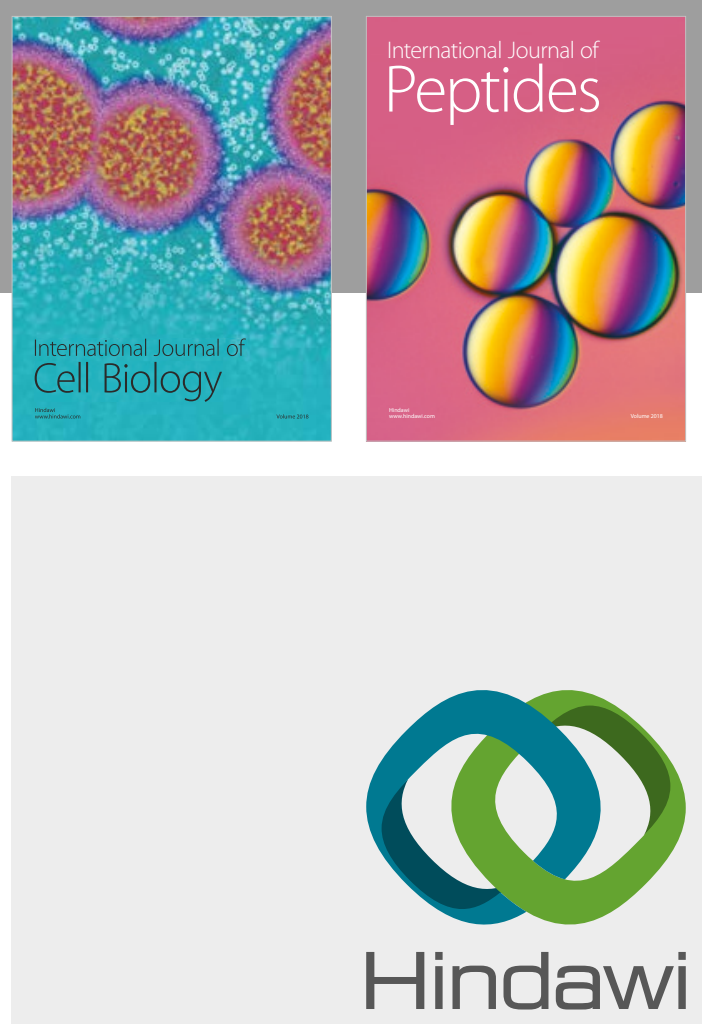

Submit your manuscripts at

www.hindawi.com
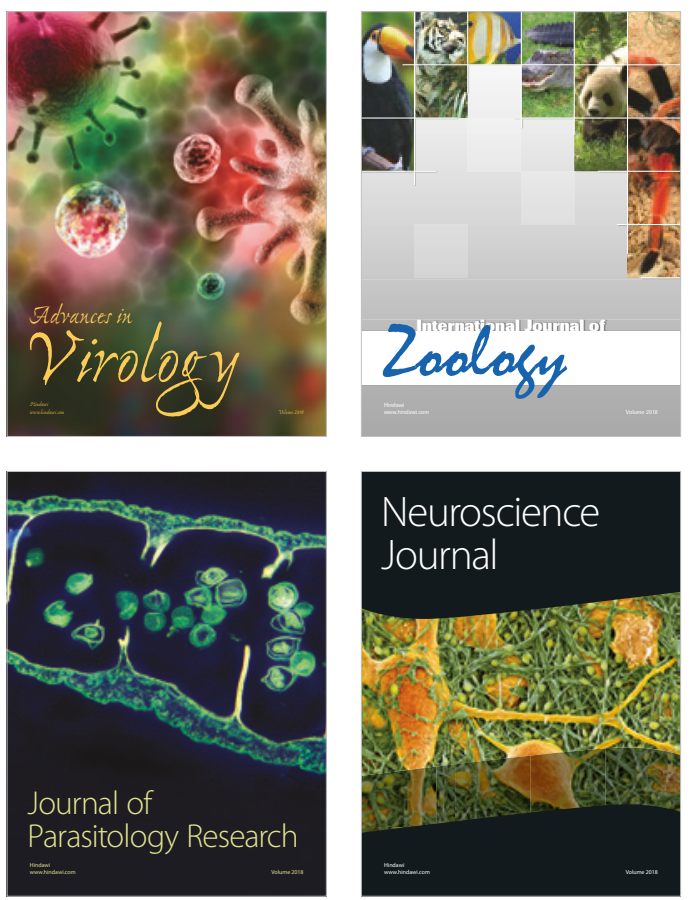
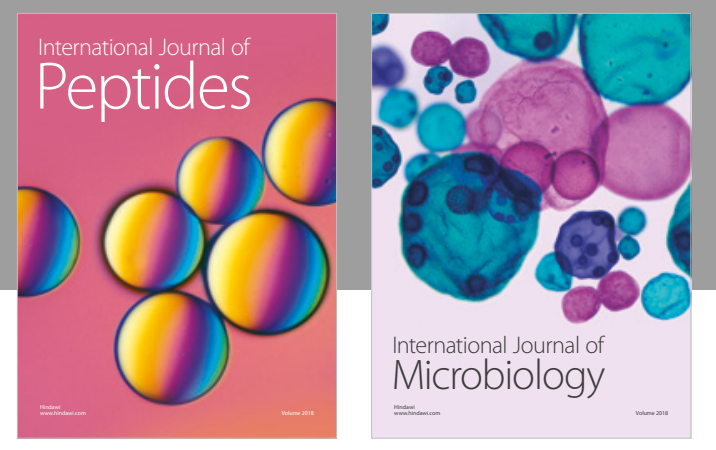

nternational Journal of Microbiology
Journal of
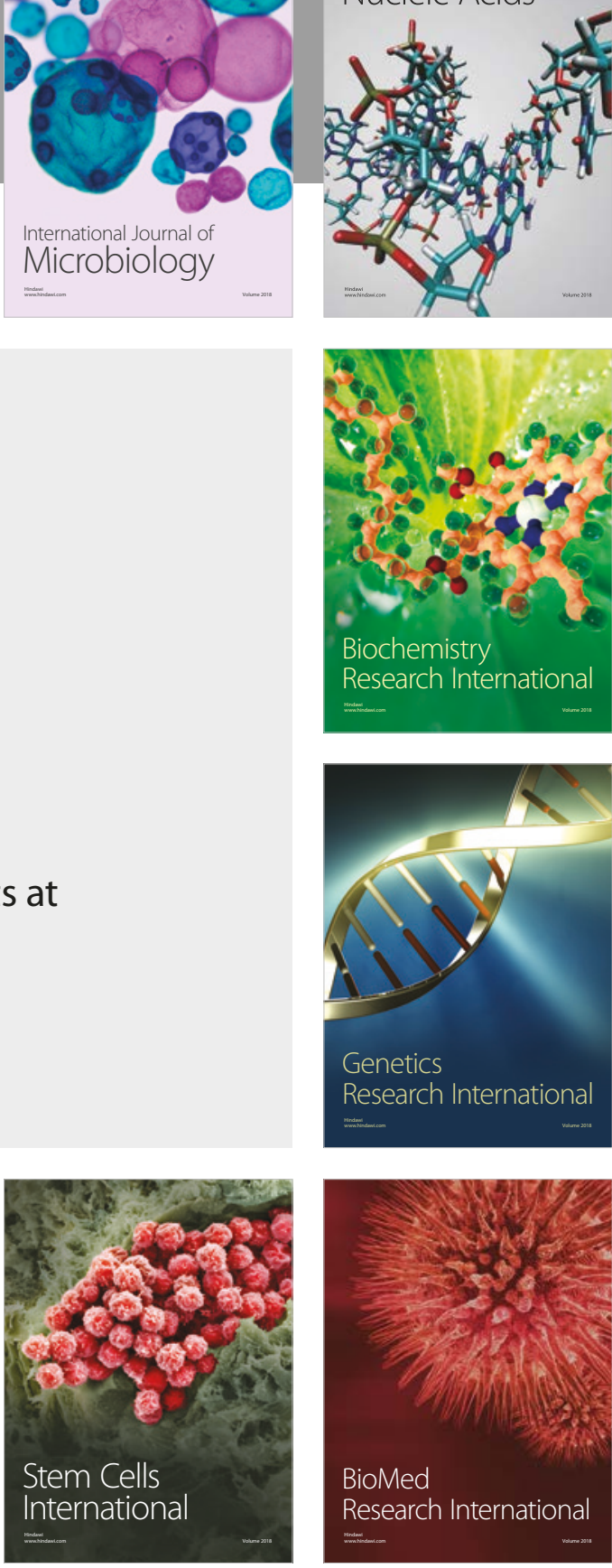
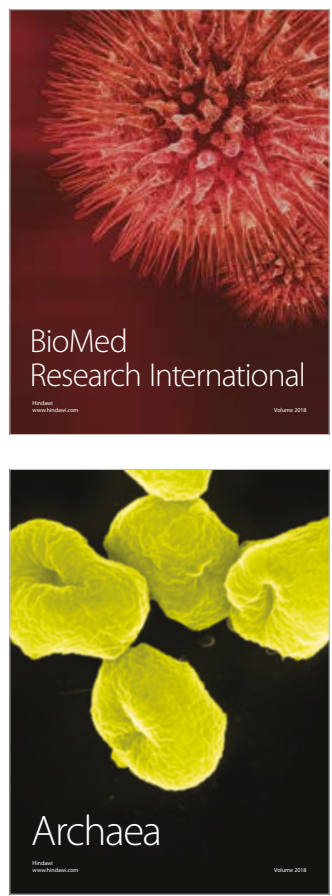